Research Article

\title{
Gene Expression Profiles of Peripheral Blood Monocytes in Osteoarthritis and Analysis of Differentially Expressed Genes
}

\author{
Ting Shi, ${ }^{1}$ Xiongjie Shen $\mathbb{D}^{2},{ }^{2}$ and Ge Gao $\mathbb{i D}^{3}$ \\ ${ }^{1}$ Department of Clinical Laboratory, Hunan Provincial People's Hospital, Changsha 410005, Hunan, China \\ ${ }^{2}$ Department of Spine Surgery, Hunan Provincial People's Hospital, Changsha 410005, Hunan, China \\ ${ }^{3}$ Department of Clinical Laboratory, Xiangya School of Medicine, Central South University, Changsha 410005, Hunan, China
}

Correspondence should be addressed to Xiongjie Shen; tianhai_521@163.com

Received 17 April 2019; Revised 27 July 2019; Accepted 22 October 2019; Published 26 November 2019

Academic Editor: Graziano Pesole

Copyright (c) 2019 Ting Shi et al. This is an open access article distributed under the Creative Commons Attribution License, which permits unrestricted use, distribution, and reproduction in any medium, provided the original work is properly cited.

\begin{abstract}
Background. There is little understanding of the molecular processes involved in the pathogenesis of osteoarthritis, limiting early diagnosis and effective treatment of OA. Use of genechips can provide insights into the molecular pathogenesis of diseases. In this study, determination of gene expression profiles of osteoarthritis peripheral blood mononuclear cells will allow exploration of the molecular pathogenesis of OA and find out more candidate biomarkers and potential drug targets of OA. Result. A total of 1231 DEGs were screened out including 791 upregulated DEGs and 440 downregulated DEGs. The most significant upregulated DEG was RPL38, which may inhibit chondrocyte differentiation and synthesis of the extracellular matrix. PIK3CA, PIK3CB, PIK3CD, PIK3R1, MAPK14, IL1A, JUND, FOSL2, and PPP3CA were the gene symbols of the osteoclast differentiation pathway which was the most significant pathway enriched by DEGs. However, the MAPK signaling pathway occupied the core position of all the pathways which can regulate apoptosis, cell cycle, wnt signaling pathway, p53 signaling pathway, and phosphatidylinositol signaling system. Furthermore, PI3Ks may regulate IL1A, JUND, FOSL2 and PPP3CA through the MAPK signaling pathway. Conclusion. These identified DEGs and pathways may be novel biomarkers to monitor the changes of OA and can be a potential drug target for the treatment of OA.
\end{abstract}

\section{Background}

Osteoarthritis $(\mathrm{OA})$ is a chronic degenerative joint disease characterized by degeneration of articular cartilage, synovium inflammation, imbalance in the synthesis and catabolism of the extracellular matrix of chondrocytes, and the formation of subchondral bone and osteophytes [1]. OA is common in the elderly, especially people older than 65 [2]. It is predominant in heavily loaded joints including the knee, hip, spine, and finger joints and ultimately leads to joint dysfunction [3]. Although there are many various therapies to relieve joint pain and improve joint function, the efficacy of these treatments is limited [4]. Joint replacement surgery can only treat patients at the end stage of OA, and X-ray diagnosis is not informative without visible radiographic changes in joint tissue. There is little understanding of the molecular processes involved in the pathogenesis of $\mathrm{OA}$, limiting early diagnosis and effective treatment of OA. Thus, the identification of sensitive biomarkers and the development of novel drug targets at molecular level are key goals of OA research.

Together with the Human Genome Project and the rapid development of molecular biology technology, highthroughput genechip technology has emerged, allowing the rapid and simultaneous analysis of thousands of gene loci [5]. Use of genechips can provide insights into the molecular pathogenesis of diseases. Currently, gene expression profiles of OA have mainly focused on articular cartilage, subchondral bone, and synovium [6-8], but there has not been comprehensive microarray analysis of blood monocytes. Blood is more accessible than tissue, and blood sample collection is less painful for patients, so the identification of sensitive diagnostic biomarkers of $\mathrm{OA}$ in the peripheral blood would be highly valuable for clinical application. 
Peripheral blood mononuclear cells (PBMCs) participate in the occurrence and development of osteoarthritis by promoting osteoclastogenesis and bone resorption, and inhibiting osteoclast apoptosis and interleukin 1 receptor I (IL-1RI) expression [9]. Monocytes increase the degradation of the extracellular matrix of chondrocytes by promoting the expression of matrix metalloproteinase 13 (MMP13), an enzyme that participates in the degradation of extracellular matrix proteins [10]. Monocytes promote the apoptosis of chondrocytes and ultimately lead to cartilage degeneration [11]. Therefore, determination of gene expression profiles of osteoarthritis PBMCs will allow exploration of the molecular pathogenesis of osteoarthritis and may help identify improved targets for the treatment of osteoarthritis.

In this study, gene expression profiles of osteoarthritis PBMCs were constructed by genechip technology. Differentially expressed genes (DEGs) were screened out by comparing the genechip results of osteoarthritis patients with those of normal controls. To obtain greater insights into the molecular mechanisms of $\mathrm{OA}$, we applied bioinformatics analysis. Gene ontology (GO) analysis and pathway enrichment analysis were performed for DEGs on the GeneCloud of Biotechnology Information (GCBI) bioinformatics platform, revealing the core genes and signaling pathways in the pathogenesis of $\mathrm{OA}$. In addition, the network relationships between DEGs and signaling pathways were determined by pathway relation and gene signal network analyses, revealing key players in the molecular pathogenesis of OA.

\section{Results}

2.1. Identification of Differentially Expressed Genes. Gene expression profiles of peripheral blood monocytes for OA groups and control groups were compared, revealing 1231 DEGs. Of these genes, 791 were upregulated and 440 were downregulated. We ranked the differentially expressed genes according to the $P$ value. The top thirty up- and downregulated DEGs are listed in Table 1 . The lowest $P$ value of the upregulated DEGs was ribosomal protein L38 (RPL38; $\left.P=5.30 \times 10^{-05}\right)$, followed by protein phosphatase 3 , catalytic subunit, alpha isozyme $\left(P P P 3 C A ; P=7.40 \times 10^{-05}\right)$. The most significant downregulated DEG was IKAROS family zinc finger 1 (IKZF1; $P=1.36 E-04)$, followed by chromosome 7 open reading frame 43 (C7orf43; $P=1.87 E-04$ ). Cluster analysis was performed on the differentially expressed genes, and the result is represented as a heatmap (Figure 1).

2.2. GO Analysis and Pathway Enrichment Analysis of DEGs. We next performed GO analysis of the identified DEGs and found 449 biological processes that were significantly enriched. The 30 most enriched GO terms and related DEGs are shown in Table 2. We found that the DEGs were mainly enriched in small molecule metabolic process (GO:0044281, GO:0006468, and GO:0044267), immune process (GO: 0045087, GO:0006955, GO:0006954, GO:0019221, and GO: 0050900), cell proliferation and apoptosis (GO:0006915, GO:
0008283, GO:0043065, and GO:0008219), cell cycle (GO: 0000278, GO:0000090, GO:0000087, and GO:0007049), RNA splicing process (GO:0008380 and GO:0000398), and cell adhesion (GO:0030155). DEGs enriched in these biological processes included interleukin 1, alpha (IL1A), interleukin 6 receptor (IL6R), interleukin 7 receptor (IL7R), protein phosphatase 3, catalytic subunit, alpha isozyme (PPP3CA), ribosomal protein L38 (RPL38), phosphatidylinositol-4,5-bisphosphate 3-kinase (PIK3), mitogen-activated protein kinase 14 (MAPK14), tumor necrosis factor superfamily, member 10 (TNFSF10), tumor necrosis factor superfamily, member 13 (TNFSF13), activating transcription factor 2 (ATF2), and others. The DEGs mapped to 143 pathways, and the pathway enrichment analysis is partly presented in Table 3. Many of the genes with altered expression participate in osteoclast differentiation, apoptosis, focal adhesion, and cell cycle. In addition, many DEGs are parts of signaling pathways like the MAPK, PI3K-Akt, calcium, T-cell receptor, and wnt and Jak-STAT signaling pathways. Tyrosine kinase 2 (TYK2), phosphatidylinositol4,5-bisphosphate 3-kinase, catalytic subunit alpha (PIK3CA), phosphatidylinositol-4,5-bisphosphate 3-kinase, catalytic subunit beta (PIK3CB), phosphatidylinositol-4,5-bisphosphate 3-kinase, catalytic subunit delta (PIK3CD), phosphoinositide-3-kinase, regulatory subunit 1 (PIK3R1), MAPK14, IL1A, jun D proto-oncogene (JUND), FOS-like antigen 2 (FOSL2), and PPP3CA genes are related to osteoclast differentiation, and $I L-1 A, J U N D, P P P 3 C A$, and $M A P K 14$ are involved in the MAPK signaling pathway.

2.3. Network Analysis. The geneSignalNetwork analysis was performed on 1231 DEGs and revealed interactions between 183 DEGs (Figure 2). The results showed that phospholipase $C$, beta 1 (PLCB1), PIK3CA, PIK3CB, PIK3CD, PIK3R1, v$K i$-ras2 Kirsten rat sarcoma viral oncogene homolog (KRAS), MAPK14, protein kinase, cAMP-dependent, catalytic, beta (PRKACB), phosphatidylinositol-4-phosphate 5-kinase, type I, alpha (PIP5K1A), G protein, alpha inhibiting activity polypeptide 2 (GNAI2), G protein, alpha inhibiting activity polypeptide 3 (GNAI3), calmodulin 1 (CALM1), G protein, beta polypeptide 2 (GNB2), G protein, gamma 11 (GNG11), beta-actin (ACTB), and Rac family small GTPase2 (RAC2) interact with more than 10 DEGs, making them core genes in the network. The network relationship of DEGs related to osteoclast differentiation, including $P I K 3 C A, P I K 3 C B$, PIK3CD, PIK3R1, MAPK14, JUND, FOSL2, IL1A, and $P P P 3 C A$, is shown in Figure 3.

The pathwayRelationNetwork analysis was also performed and revealed interaction relationships between 68 pathways (Figure 4). The MAPK signaling pathway, apoptosis, pathways in cancer, cell cycle, p53 signaling pathway, calcium signaling pathway, wnt signaling pathway, adherens junction, ErbB signaling pathway, focal adhesion, regulation of actin cytoskeleton, ubiquitin-mediated proteolysis, TGFbeta signaling pathway, and pancreatic cancer interacted with more than 10 pathways, making these core pathways. The most significant pathway in this analysis was the MAPK signaling pathway and its related pathways, as shown in 
TABLE 1: The top 30 up- and downregulated DEGs in peripheral blood mononuclear cells of patients with OA.

\begin{tabular}{|c|c|c|c|}
\hline Gene symbol upregulated expression & Accession number & $P$ value & Fold change \\
\hline RPL38 & NM_000999 & $5.30 E-05$ & 1.70 \\
\hline PPP3CA & NM_000944 & $7.40 E-05$ & 1.42 \\
\hline TROVE2 & NM_001042369 & $8.10 E-05$ & 1.28 \\
\hline PRPF40A & NM̄M_017892 & $9.50 E-05$ & 1.41 \\
\hline CEP350 & NM_014810 & $1.18 E-04$ & 1.52 \\
\hline TROVE2 & NM_001042369 & $1.47 E-04$ & 1.35 \\
\hline CEP350 & NM_014810 & $1.59 E-04$ & 1.41 \\
\hline MRPS31 & NM_005830 & $1.65 E-04$ & 1.33 \\
\hline RAB11A & NM_001206836 & $1.84 E-04$ & 1.44 \\
\hline ZC3H14 & NM_001160103 & $1.85 E-04$ & 1.35 \\
\hline USP12 & NM_182488 & $1.96 E-04$ & 1.38 \\
\hline GTF2H5 & NM_207118 & $2.32 E-04$ & 1.37 \\
\hline ITPR1 & NM_001099952 & $2.45 E-04$ & 1.35 \\
\hline MTF2 & NM_001164391 & $2.54 E-04$ & 1.44 \\
\hline CLCN3 & NM_001243372 & $2.59 E-04$ & 1.31 \\
\hline C6orf62 & NM̄_030939 & $2.94 E-04$ & 1.56 \\
\hline MOSPD1 & NM_019556 & $3.25 E-04$ & 1.25 \\
\hline PIGF & NM_002643 & $3.54 E-04$ & 1.43 \\
\hline PREPL & NM_001042385 & $3.89 E-04$ & 1.39 \\
\hline THUMPD1 & NM_017736 & $4.08 E-04$ & 1.37 \\
\hline CEP57 & NM_001243776 & $4.13 E-04$ & 1.41 \\
\hline PHACTR2 & NM_001100164 & $4.41 E-04$ & 1.43 \\
\hline ADAMTS10 & NM_-001110 & $4.52 E-04$ & 1.28 \\
\hline E2F3 & NM_001243076 & $4.53 E-04$ & 1.41 \\
\hline ING3 & NM_019071 & $4.71 E-04$ & 1.49 \\
\hline MYCBP2 & NM_015057 & $4.75 E-04$ & 1.32 \\
\hline USP7 & NM_003470 & $4.90 E-04$ & 1.39 \\
\hline PHKB & NM_000293 & $5.07 E-04$ & 1.28 \\
\hline MED1 & NM_004774 & $5.19 E-04$ & 1.41 \\
\hline NDUFA5 & NM_005000 & $5.78 E-04$ & 1.51 \\
\hline IKZF1 & NM_001220765 & $1.36 E-04$ & 1.56 \\
\hline C7orf43 & NM_018275 & $1.87 E-04$ & 1.29 \\
\hline RAB3D & NM_004283 & $2.51 E-04$ & 1.33 \\
\hline CDV3 & NM_001134422 & $2.59 E-04$ & 1.25 \\
\hline FRS3 & NM_006653 & $2.64 E-04$ & 1.23 \\
\hline ARF3 & NM_001659 & $3.46 E-04$ & 1.34 \\
\hline LMO4 & NM_006769 & $3.64 E-04$ & 1.22 \\
\hline RING1 & NM_002931 & $4.61 E-04$ & 1.23 \\
\hline NADK & NM_001198993 & $5.82 E-04$ & 1.36 \\
\hline MTMR3 & NM_001013676 & $5.99 E-04$ & 1.28 \\
\hline PTGES2 & NM_001256335 & $6.04 E-04$ & 1.34 \\
\hline MAX & NM_001271068 & $6.32 E-04$ & 1.47 \\
\hline LOC100996752 & NM_019094 & $6.73 E-04$ & 1.22 \\
\hline MARS & NM_004990 & $8.23 E-04$ & 1.21 \\
\hline SIGLEC7 & NM_001277201 & $8.49 E-04$ & 1.24 \\
\hline DUSP3 & NM_004090 & $8.91 E-04$ & 1.39 \\
\hline TNFAIP2 & NM_006291 & $9.11 E-04$ & 1.38 \\
\hline MAT2A & NM_005911 & $9.78 E-04$ & 1.41 \\
\hline BRD2 & NM_001113182 & $1.06 E-03$ & 1.44 \\
\hline MED12 & NM__005120 & $1.23 E-03$ & 1.23 \\
\hline SLC25A28 & NM_031212 & $1.26 E-03$ & 1.24 \\
\hline STX16 & NM_001001433 & $1.44 E-03$ & 1.39 \\
\hline USP21 & NM_001014443 & $1.50 E-03$ & 1.26 \\
\hline TM9SF4 & NM_014742 & $1.55 E-03$ & 1.39 \\
\hline $\mathrm{CBFB}$ & NM_001755 & $1.57 E-03$ & 1.28 \\
\hline GGA2 & NM_015044 & $1.60 E-03$ & 1.21 \\
\hline POLG & NM_001126131 & $1.61 E-03$ & 1.34 \\
\hline YKT6 & NM_006555 & $1.68 E-03$ & 1.25 \\
\hline CLASRP & NM_001278439 & $1.75 E-03$ & 1.28 \\
\hline RCN3 & NM̄_020650 & $1.94 E-03$ & 1.21 \\
\hline
\end{tabular}




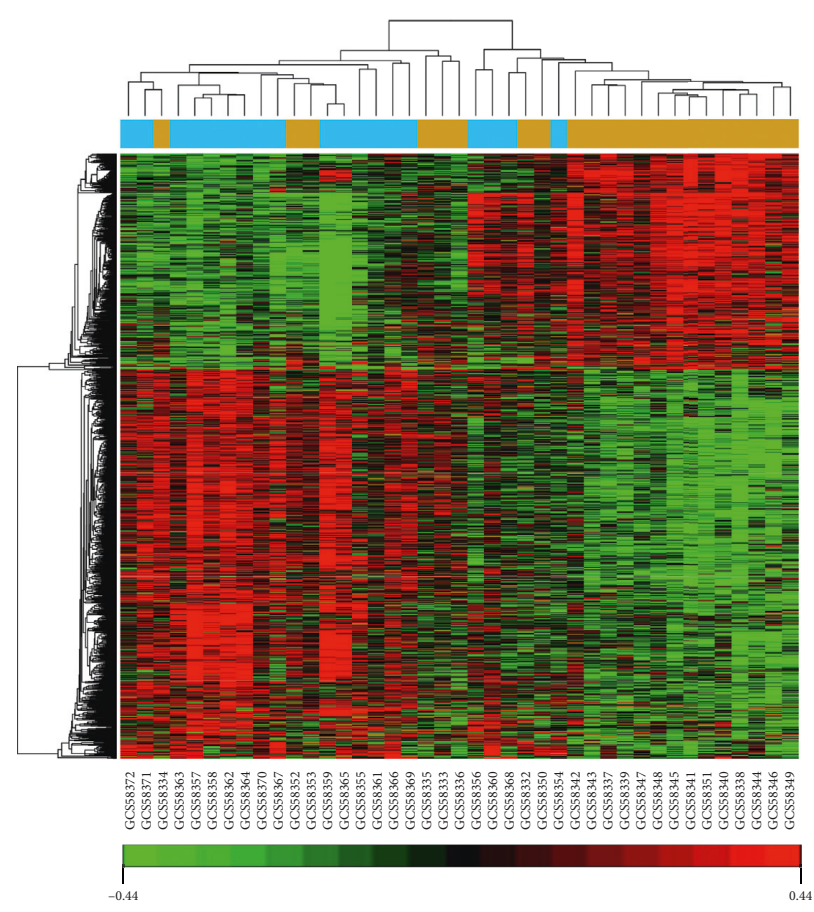

FIgURE 1: A heatmap. The horizontal axis indicated cluster analysis of groups. Blue represented OA groups, and brown represented control groups. The vertical axis meant cluster analysis of genes. Red represented upregulated DEGs, green represented downregulated DEGs, and black represented the genes expression with no difference. The more the significant difference of gene expression, the brighter the color.

TABle 2: The top 30 GO terms.

\begin{tabular}{|c|c|c|c|c|c|}
\hline GO ID & GO name & $\begin{array}{c}\text { DEG } \\
\text { number }\end{array}$ & $P$ value & FDR & Gene symbols \\
\hline GO:0044281 & $\begin{array}{l}\text { Small molecule metabolic } \\
\text { process }\end{array}$ & 121 & $1.65 E-34$ & $4.62 E-31$ & $\begin{array}{c}\text { PPP1CB|PLCB1|CALM1|PIK3CD| } \\
\text { PIK3R1|PIK3CA|PTEN|LYPLA1| } \\
\text { AMD1|SACM1L|ARF3| INSIG1| } \\
\text { PDK3|ABHD5|CHKB|IMPA1,... } \\
\text { IL7R|TNFSF12|TNFSF10|TNFSF4| }\end{array}$ \\
\hline GO:0007165 & Signal transduction & 100 & $1.19 E-31$ & $1.66 E-28$ & $\begin{array}{l}\text { PPP2R5C|PLCB1|CALM1|PIK3CB| } \\
\text { PIK3CD|CASP1| TLR1| RAC2| } \\
\text { STAT6|MAPK14|CAMK1|PAK2,... } \\
\text { CASP1|ATF1|PIK3CA|PIK3R1| }\end{array}$ \\
\hline GO:0045087 & Innate immune response & 64 & $1.57 E-24$ & $1.47 E-21$ & $\begin{array}{c}\text { MAP3K7|MAP3K5|KRAS|TLR1| } \\
\text { PTEN| }\end{array}$ \\
\hline \multicolumn{6}{|l|}{$\begin{array}{l}\text { ATF2|CFP|PTPN11| IRF7| } \\
\text { ACTB|CALM1,... }\end{array}$} \\
\hline GO:0010467 & Gene expression & 69 & $1.53 E-23$ & $1.07 E-20$ & $\begin{array}{c}\text { TNFSF13|PPP2R1A|RPL38| } \\
\text { MAPK14|KARS|SMAD2|AIMP1| } \\
\text { DICER1,... }\end{array}$ \\
\hline GO:0006915 & Apoptotic process & 66 & $5.32 E-22$ & $2.98 E-19$ & $\begin{array}{c}\text { IL1A|TNFSF10|TNFSF12|MAP3K7| } \\
\text { CASP1|BAX|RAD21|DICER1| } \\
\text { CASP4|PTEN|H1F0|FXR1|PSMD12| } \\
\text { DAB2|CST3,... }\end{array}$ \\
\hline GO:0000278 & Mitotic cell cycle & 47 & $2.64 E-20$ & $1.23 E-17$ & $\begin{array}{c}\text { PPP1R12A|PPP1CB|PPP2R1A|NSL1| } \\
\text { RFC5|RAD21|RBL2|RPA1|CEP63... } \\
\text { MAP3K7|ATF1|ATF6|STAT6| }\end{array}$ \\
\hline GO:0006351 & $\begin{array}{l}\text { Transcription, DNA- } \\
\text { dependent }\end{array}$ & 114 & $1.32 E-19$ & $5.28 E-17$ & $\begin{array}{c}\text { FOSL2|ASF1A|BBX|UCHL5| } \\
\text { ATXN1|INO80 B|KANK2|CEBPE| } \\
\text { SIRT1| SETD2|IRF5| BRD2|IKZF1| } \\
\text { RB1,... }\end{array}$ \\
\hline GO:0019221 & $\begin{array}{l}\text { Cytokine-mediated } \\
\text { signaling pathway }\end{array}$ & 27 & $1.63 E-19$ & $5.70 E-17$ & $\begin{array}{c}\text { IL1A|IL6R|STAT2|STAT6|KRAS| } \\
\text { CEBPA|PTPN11|CD44|IRF7|IRF5| } \\
\text { IRF3|IRAK3|ZC3H15|CCR1,... }\end{array}$ \\
\hline
\end{tabular}


TABle 2: Continued.

\begin{tabular}{|c|c|c|c|c|c|}
\hline GO ID & GO name & $\begin{array}{c}\text { DEG } \\
\text { number }\end{array}$ & $P$ value & FDR & Gene symbols \\
\hline GO:0019048 & Virus-host interaction & 42 & $4.74 E-19$ & $1.48 E-16$ & $\begin{array}{c}\text { MAP3K5|PIK3R1|KARS|BAX| } \\
\text { TGFB1|CCR3|MDFIC| RB1| } \\
\text { SIRT1,... }\end{array}$ \\
\hline GO:0006468 & Protein phosphorylation & 43 & $2.25 E-18$ & $6.30 E-16$ & $\begin{array}{c}\text { MAP3K11|MAP4K4|PIK3R1| } \\
\text { PIK3CA|TGFB1|ADAM10|IRAK3| } \\
\text { HIPK3|SMAD2| TLK1|CREB1|FYB| } \\
\text { STK11,... }\end{array}$ \\
\hline GO:0008380 & RNA splicing & 34 & $3.49 E-16$ & $7.52 E-14$ & $\begin{array}{c}\text { PPP2R1A|SRSF11|PABPN1|CPSF1| } \\
\text { SRSF3|CLASRP,... }\end{array}$ \\
\hline GO:0015031 & Protein transport & 43 & $3.87 E-16$ & $7.73 E-14$ & $\begin{array}{c}\text { RAB3D|ARRB2|RAB11A|ACAP1| } \\
\text { NMD3|ARF3,... }\end{array}$ \\
\hline GO:0044267 & $\begin{array}{l}\text { Cellular protein metabolic } \\
\text { process }\end{array}$ & 45 & $4.71 E-13$ & $6.94 E-11$ & $\begin{array}{c}\text { ATF1|RPL38|ACTB|ATF6|CPA3| } \\
\text { EIF4B|ETF1|SPCS2 } \mid \text { CXXC1|ALG5 } \mid \\
\text { CCT6A,... }\end{array}$ \\
\hline GO:0000090 & Mitotic anaphase & 24 & $4.61 E-12$ & $5.87 E-10$ & $\begin{array}{c}\text { PPP2R1A|RAD21|PDS5A|PSMD3| } \\
\text { SMC3|CLASP2, . . }\end{array}$ \\
\hline GO:0000087 & M phase of mitotic cell cycle & 26 & $7.27 E-12$ & $8.85 E-10$ & $\begin{array}{l}\text { PPP2R1A|PSMD4|CLASP2|PSME3| } \\
\text { ANAPC5|RAD21|RANBP2,... }\end{array}$ \\
\hline GO:0000398 & $\begin{array}{l}\text { mRNA splicing via } \\
\text { spliceosome }\end{array}$ & 24 & $7.84 E-12$ & $9.15 E-10$ & $\begin{array}{c}\text { SRSF11|PABPN1|CLP1|RBM22| } \\
\text { PAPOLA|FUS|POLR2E|TRA2A| } \\
\text { PRPF4B,... }\end{array}$ \\
\hline GO:0001525 & Angiogenesis & 24 & $9.13 E-12$ & $1.02 E-09$ & $\begin{array}{c}\text { TNFSF12|MAP3K7|MAPK14| } \\
\text { PIK3CA|ELK3|DICER1|SIRT1,... } \\
\text { MAP3K7|MAPK14|TLR7|IRF7| }\end{array}$ \\
\hline GO:0002224 & $\begin{array}{l}\text { Toll-like receptor signaling } \\
\text { pathway }\end{array}$ & 19 & $3.87 E-11$ & $3.87 E-09$ & $\begin{array}{c}\text { ATF1|ATF2|DUSP3|IRF3|HSP90B1| } \\
\text { RPS6KA5|BTK| UNC93B1|ELK1| } \\
\text { BCL10|UBE2D1,... }\end{array}$ \\
\hline GO:0007049 & Cell cycle & 24 & $4.71 E-11$ & $4.55 E-09$ & $\begin{array}{c}\text { PPP1CA|CAMK1|RB1CC1|CREBL2| } \\
\text { PARD6A|CYLD|TLK1| IKZF1,... } \\
\text { IL1A|IL7R|IL1RN|TNFSF10| }\end{array}$ \\
\hline GO:0006955 & Immune response & 29 & $7.21 E-11$ & $6.73 E-09$ & $\begin{array}{c}\text { TNFSF4|TNFSF13|TLR1|CCR1| } \\
\text { IKBKAP, . }\end{array}$ \\
\hline GO:0008283 & Cell proliferation & 29 & $5.42 E-10$ & $4.27 E-08$ & $\begin{array}{c}\text { IL1A|RBL38|MAP3K11|PTEN| } \\
\text { DAB2|FES|PA2G4|ASCC3| } \\
\text { MS4A2,... }\end{array}$ \\
\hline GO:0030155 & Regulation of cell adhesion & 10 & $5.49 E-10$ & $4.27 E-08$ & $\begin{array}{c}\text { PPP2R1A|PPP1R12 A|PPP1CB| } \\
\text { CYTH4|PTK2B| CYTIP| ENG| } \\
\text { GSN,... }\end{array}$ \\
\hline GO:0050852 & $\begin{array}{l}\text { T-cell receptor signaling } \\
\text { pathway }\end{array}$ & 16 & $6.55 E-10$ & $4.96 E-08$ & $\begin{array}{c}\text { MAP3K7|PIK3CA|PIK3CB|PIK3R1| } \\
\text { PIK3CD|RBCK1|PAK2|FYB|UBE2N| } \\
\text { PTEN|WAS|FYN|NCK1|MALT1| } \\
\text { CD4| BCL1 }\end{array}$ \\
\hline GO:0043065 & $\begin{array}{l}\text { Positive regulation of } \\
\text { apoptotic process }\end{array}$ & 20 & $8.03 E-10$ & $5.92 E-08$ & $\begin{array}{c}\text { TNFSF10|PPP2R4|MAP3K5|PIK3R1| } \\
\text { TGFB1|ATM|SIRT1|DNM2|LILRB1| } \\
\text { CTNNB1|TSPO|HMGB1|BAX| } \\
\text { ING3|IRF5|PRKDC,... }\end{array}$ \\
\hline GO:0051403 & $\begin{array}{l}\text { Stress-activated MAPK } \\
\text { cascade }\end{array}$ & 10 & $1.92 E-09$ & $1.38 E-07$ & $\begin{array}{c}\text { MAPK14|MAP3K7|ATF1|ATF2| } \\
\text { ELK1|DUSP3|RPS6KA3|CREB1| } \\
\text { RPS6KA1|RPS6KA5 }\end{array}$ \\
\hline GO:0007010 & Cytoskeleton organization & 10 & $4.27 E-09$ & $2.49 E-07$ & $\begin{array}{l}\text { PRPF40 A|PHIP|SIPA1|PLD2|SVIL| } \\
\text { PXN|TPM1|ZMYM4|CAPZB,... }\end{array}$ \\
\hline GO:0050900 & Leukocyte migration & 14 & $6.19 E-09$ & $3.54 E-07$ & $\begin{array}{l}\text { PIK3CB| PIK3CA|PIK3R1|KRAS| } \\
\text { SIRPA| PTPN11|FYN|ITGB7,... } \\
\text { IL1A|PIK3CD|TLR1|TLR7|STAB1| }\end{array}$ \\
\hline GO:0006954 & Inflammatory response & 19 & $1.11 E-08$ & $6.21 E-07$ & $\begin{array}{c}\text { TGFB1|HRH4|CCR3|CCR1| } \\
\text { LTB4R, . . }\end{array}$ \\
\hline GO:0008219 & Cell death & 16 & $8.44 E-08$ & $3.94 E-06$ & $\begin{array}{c}\text { MAP3K11|BCL10|FOSL2|CLN3| } \\
\text { ATXN7|DCTN1|FUS|,... }\end{array}$ \\
\hline GO:0006281 & DNA repair & 30 & $9.06 E-08$ & $4.16 E-06$ & $\begin{array}{c}\text { BTG2|RFC5|SMC3|RPA1|TDG| } \\
\text { CSNK1D } \mid \text { REV1,... }\end{array}$ \\
\hline
\end{tabular}


TABLE 3: Results of pathway enrichment analysis of DEGs.

\begin{tabular}{|c|c|c|c|}
\hline Pathway name & DEG number & $P$ value & Gene symbols \\
\hline Osteoclast differentiation & 26 & $6.26 E-16$ & $\begin{array}{c}\text { TYK2|PIK3CA|PIK3CB } \mid \text { PIK3CD|PIK3R1|IL1A } \mid \\
\text { PPP3CA|JUND|FOSL2|MAPK14|MAP3K7|STAT2 } \mid \\
\text { TGFB1,... }\end{array}$ \\
\hline Metabolic pathways & 79 & $2.81 E-15$ & COX11|PIP5K1A|PLCB1|CHPF2|ALOX5|TPK1,... \\
\hline PI3K-Akt signaling pathway & 34 & $1.77 E-11$ & $\begin{array}{c}\text { IL7R|IL6R|PPP2R5C|PPP2R1A|PIK3CB|PIK3CD } \mid \\
\text { PIK3CA|ATF2,... }\end{array}$ \\
\hline MAPK signaling pathway & 28 & $1.34 E-10$ & $\begin{array}{c}\text { IL1A|PPP3CA|JUND|MAPK14|MAP3K5|MAP3K7| } \\
\text { KRAS|TGFB1,.. }\end{array}$ \\
\hline Chemokine signaling pathway & 24 & $1.39 E-10$ & $\begin{array}{c}\text { PLCB1 } \mid \text { CCR1 } \mid \text { CCR3|PIK3R1|PIK3CD|PIK3CB } \mid \\
\text { PIK3CA|KRAS|RAC2|STAT2,... }\end{array}$ \\
\hline T-cell receptor signaling pathway & 17 & $2.29 E-09$ & $\begin{array}{c}\text { PPP3CA|MAPK14|MAP3K7|PIK3CD|PIK3CB } \mid \\
\text { PIK3R1|PIK3CA|KRAS|BCL10,... }\end{array}$ \\
\hline Focal adhesion & 23 & $3.28 E-09$ & $\begin{array}{c}\text { PPP1CA } \mid \text { PPP1CB } \mid \text { ACTB } \mid \text { PIK3CA } \mid \text { PIK3CD } \mid \text { PIK3CB } \mid \\
\text { PTEN, ... }\end{array}$ \\
\hline Apoptosis & 15 & $6.96 E-09$ & $\begin{array}{c}\text { IL1A|TNFSF10|TNFRSF10B|PIK3CD } \mid \text { PIK3CB } \mid \\
\text { PIK3CA|BAX|IRAK3,... }\end{array}$ \\
\hline Pathways in cancer & 27 & $9.27 E-08$ & $\begin{array}{c}\text { PIK3CA|PIK3CB } \mid \text { PIK3R1|PIK3CD } \mid \text { KRAS } \mid \text { PTEN } \mid \\
\text { MAX|RAC2,... }\end{array}$ \\
\hline Cell cycle & 16 & $1.29 E-07$ & ANAPC5|TGFB1|SMAD2|ATM|RBL2|RB1,... \\
\hline VEGF signaling pathway & 11 & $1.26 E-06$ & $\begin{array}{c}\text { PPP3CA|MAPK14|PIK3CA|PIK3CD|PIK3R1| } \\
\text { PIK3CB|RAC2|KRAS, . . }\end{array}$ \\
\hline Toll-like receptor signaling pathway & 11 & $1.37 E-04$ & $\begin{array}{c}\text { MAPK14|MAP3K7|PIK3CD|PIK3R1|PIK3CA| } \\
\text { PIK3CB|TLR7|TLR1|IRF5|IRF7, . . }\end{array}$ \\
\hline Calcium signaling pathway & 14 & $3.47 E-04$ & PPP3CA|PLCB1|CALM1|ITPR1|ITPR2|PTK2B,... \\
\hline p53 signaling pathway & 8 & $4.91 E-04$ & $\begin{array}{c}\text { TNFRSF10B } \mid \text { ATM|PTEN|THBS1|BAX } \mid \text { CCNG2| } \\
\text { CCNG1,... }\end{array}$ \\
\hline Wnt signaling pathway & 10 & $5.49 E-03$ & PPP3CA|MAP3K7|PLCB1|RAC2|CHP|, . . \\
\hline TGF- $\beta$ signaling pathway & 7 & $7.29 E-03$ & PPP2R1A|TGFB1|SMAD2|SMURF2,... \\
\hline Jak-STAT signaling pathway & 10 & $1.11 E-02$ & $\begin{array}{c}\text { IL7R|IL6R|PIK3R1|PIK3CA|PIK3CD|PIK3CB| } \\
\text { STAT2|STAT6,... }\end{array}$ \\
\hline
\end{tabular}

Figure 5. The calcium signaling pathway, cell cycle, wnt signaling pathway, TGF-beta signaling pathway, and VEGF signaling pathway were the source pathways of MAPK signaling pathway. Moreover, MAPK signaling pathway regulated apoptosis, cell cycle, wnt signaling pathway, p53 signaling pathway, and phosphatidylinositol signaling system.

\section{Discussion}

In the present study, the Human Genome U133A genechip from Affymetrix was used to detect the gene expression profiles of PBMCs from 19 patients with osteoarthritis and 22 healthy controls. We identified 1231 DEGs, with 791 upregulated DEGs and 440 downregulated DEGs. The top 10 most significant DEGs according to the $p$ value were RPL38, PPP3CA, TROVE domain family, member 2 (TROVE2), PRP40 pre-mRNA processing factor 40 homolog $A$ (PRPF40A), centrosomal protein $350 \mathrm{kDa}$ (CEP350), IKAROS family zinc finger 1 (IKZF1), mitochondrial ribosomal protein S31 (MRPS31), RAB11A, member RAS oncogene family $(R A B 11 A)$, zinc finger $\mathrm{CCCH}$-type containing 14 ( $\mathrm{ZC} 3 \mathrm{H} 14)$, and chromosome 7 open reading frame 43 (C7orf43). The most significant upregulated DEG was RPL38, which affects processes of gene expression, DNA transcription, gene translation, and cell proliferation. Normal expression of ribosomal protein L29 (RPL29) is essential for chondrocyte proliferation and skeletal growth, and high expression level of RPL29 inhibited chondrocyte terminal differentiation, keeping cells in a state of abnormal proliferation [12]. Altered expression of RPL29 also influenced the rate of extracellular matrix protein synthesis [13]. Green et al. found different expressions of ribosomal protein L10 (RPL10) during endochondral bone development, suggesting effects on cell differentiation before bone mineralization [14]. Given effects of other ribosomal proteins, we hypothesize that ribosomal protein RPL38 may participate in the pathogenesis of OA by inhibiting chondrocyte differentiation and proliferation, decreasing the synthesis of the extracellular matrix.

Osteoclasts are closely related to bone resorption and subchondral bone remodeling processes involved in the pathophysiology of OA [15]. Osteoclasts are large multinucleated cells which originate from hematopoietic precursors of the monocyte-macrophage lineage [16]. Durand et al. found PBMCs from OA patients display increased osteoclastogenesis and bone resorption [9]. Our pathway enrichment analysis of DEGs support previous findings and suggest that the osteoclast differentiation pathway contributes to OA development. PIK3CA, PIK3CB, PIK3CD, PIK3R1, MAPK14, IL1A, JUND, FOSL2, and PPP3CA were the gene symbols of osteoclast differentiation pathway, and these genes showed increased expression in PBMCs of OA patients compared to normal controls. This suggests that 


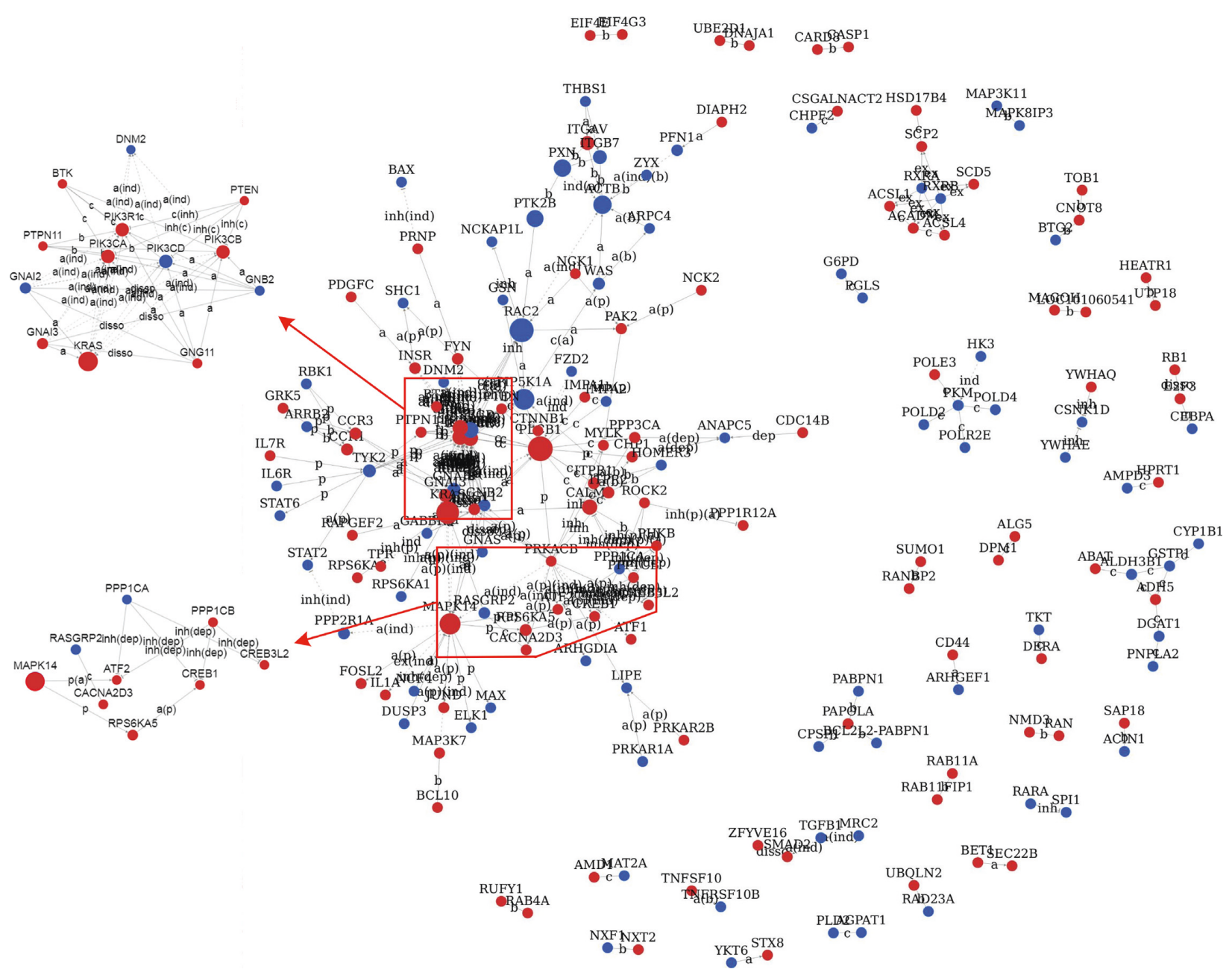

FIGURE 2: The map of DEG interaction relationship. Red nodes indicate upregulated genes, and blue nodes indicate downregulated genes. The larger the area of the node, the more important the gene in the network. The interaction relationship between genes is expressed by a connection line. The solid line without arrow indicates the interaction between DEGs is not directional, otherwise is directional. The beginning of arrow is the upstream gene, and the end of arrow is the downstream gene. The dashed line with arrow indicates the indirect interaction relationship. The solid line with flat head indicates the inhibitory interaction. The letters on the connection line are abbreviated as the type of interaction. c, compound; a, active; ind, indirect; inh, inhibition.

mononuclear cells from patients with $\mathrm{OA}$ have stronger osteoclast differentiation ability. PIK3CA, PIK3CB, PIK3CD, and PIK3R1 belong to the PI3Ks (phosphoinositide-3-kinases) family [17]. PI3K is not only involved in osteoclast differentiation, activation, and survival but also contributes to osteoclast-mediated bone resorption and in vivo bone homeostasis [18]. PI3K inhibitors such as wortmannin and LY294002 inhibit osteoclast chemotaxis, attachment, and spreading [19]. The PI3K isoform PIK3CB also promotes osteoclast development and bone resorption [20]. JUND and FOSL2 belong to the activator protein-1 (AP-1) transcriptional factor family, which regulates many cellular processes such as differentiation, proliferation, and apoptosis [21]. Fos is a necessary factor for the differentiation of hematopoietic precursor cells into osteoclasts. Mice deficient in Fos (Fos-/-) develop osteopetrosis and show high bone mineral density and defective osteoclast formation. Fos1 transgenic mice showed increased production of osteoclasts and active bone resorption [22]. The JUND/FOSL2 heterodimer upregulates the expression of Tcirg1 to increase osteoclastogenesis [23]. IL-1A, as a proinflammatory cytokine, can disrupt bone metastasis and stimulate osteoclast resorption activity [24]. PPP3CA, or calcineurin 1, is a serine/threonine-specific phosphatase regulated by $\mathrm{Ca}^{2+}$ / calmodulin [25]. PPP3C has effects in tumors, cardiovascular system, immune system, and nervous system [26]. The dephosphorylation of PPP3C helps regulate cell proliferation and differentiation in the cytokine-mediated activation of immune cells [27]. Collectively, all these upregulated DEGs associated with osteoclast differentiation may promote the development of $\mathrm{OA}$ and have potential to serve as novel biomarkers to monitor changes of $\mathrm{OA}$ and are potential drug targets for the treatment of OA.

The most significant pathway revealed by network analysis was the MAPK signaling pathway, with an occupied core position in the network. The MAPK signaling pathway as the source pathway can regulate apoptosis, cell cycle, wnt signaling pathway, p53 signaling pathway, and the 


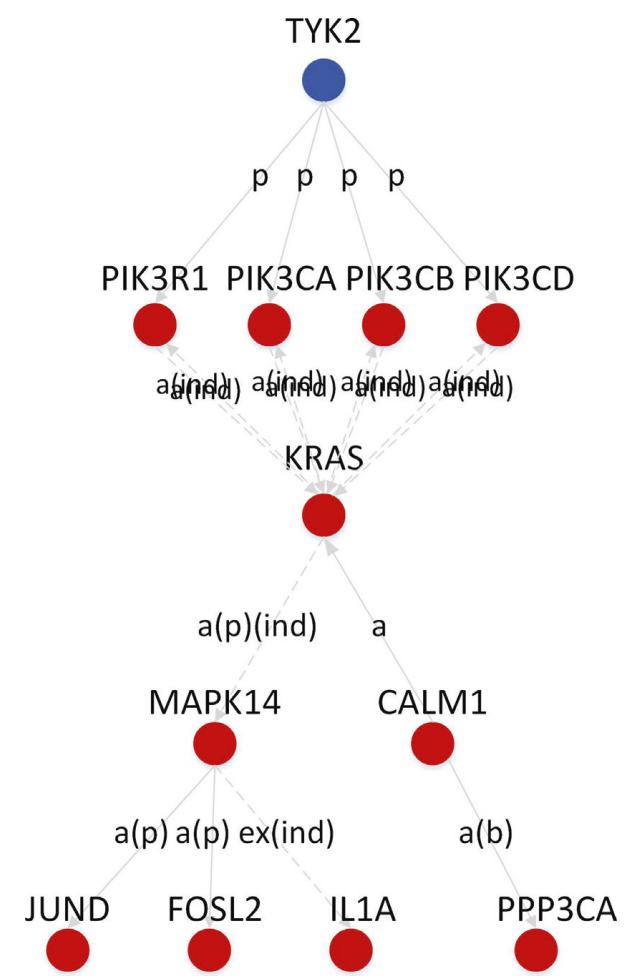

FIGURE 3: The network relationship of DEGs which enriched in osteoclast differentiation.

phosphatidylinositol signaling system. MAPKs are serine/ threonine protein kinases, and activating a protein kinase cascade (MAPKKK-MAPKK-MAPK) allows the transduction of extracellular signals into the nucleus. In this way, the expression of genes related to cell proliferation, differentiation, apoptosis, and stress response can be regulated [28]. The MAPK signaling pathway plays a variety of roles in OA. First, p38-MAPK promotes chondrocyte apoptosis through Fas-mediated apoptotic pathway and activation of ATF-2, caspase-3, and p53 [29]. Second, p38-MAPK induces the expression of MMP13, promotes the degradation of collagen II in the extracellular matrix of chondrocytes, and inhibits the synthesis and secretion of collagen and glycoprotein [30]. Third, p38-MAPK upregulates expression of cyclooxygenase-2 (COX2), prostaglandin E2 (PGE2), and inducible nitric oxide synthase (iNOS), and increases the synthesis of inflammatory mediators, leading to joint swelling, pain, and cartilage destruction [31]. Fourth, p38MAPK is essential for the differentiation of osteoclast precursor cells into osteoclasts as well as osteoclast maturation and survival [32]. In our analysis of DEGs enriched in the osteoclast differentiation pathway, we found that PIK3CA, PIK3CB, PIK3CD, and PIK3R1 indirectly activate MAPK14, the prototypic member of the p38-MAPK family, through phosphorylation. MAPK14 is a key upstream gene that activates the expression of downstream genes such as IL1A, JUND, FOSL2, and PPP3CA. The network relationships between these genes revealed that PI3Ks may regulate IL1A, JUND, FOSL2, and PPP3CA through the MAPK signaling pathway. However, these regulatory relationships require further experiment to verify.
The results of this study have similarities and differences with published microarray analysis on synovial fluid, synovium membranes, subchondral bone, and cartilage. Firstly, the biological processes enriched by some DEGs are the same, such as immune response and inflammatory response (IL, TNF, TLR, and TGF- $\beta$ ), cell cycle (TGF- $\beta$ and SMAD2), cell apoptosis (CASP and BAX). Secondly, high expression of proteinase (ADAMTS10) is same. Thirdly, some signal pathways enriched by DEGs are the same (MAPK signaling pathway, TOLL-like signaling pathway, and TGF- $\beta$ signaling pathway) [6-8]. However, this study found that the main signal pathway enriched by DEGs was osteoclast differentiation, which may be related to the fact that peripheral blood mononuclear cells are precursors of osteoclasts. Only a small portion of the DEGs have the same results as previous tissue microarray because gene expression of PBMCs is affected by several processes. Attur et al. [33] showed that PBMCs can be activated by the inflammatory process of osteoarthritis when they pass through different tissues of the joint. DEGs in PBMCs may reflect the susceptibility of OA from a genetic perspective.

\section{Conclusions}

Gene expression profiles were determined for PBMCs in OA patients and healthy controls. Analysis revealed 1231 DEGs, with 791 upregulated DEGs and 440 downregulated DEGs. The most significant upregulated DEG was RPL38, which may inhibit chondrocyte differentiation and synthesis of the extracellular matrix. PIK3CA, PIK3CB, PIK3CD, PIK3R1, MAPK14, IL1A, JUND, FOSL2, and PPP3CA were the gene symbols of the osteoclast differentiation pathway. These genes promote the development of OA through increasing osteoclastogenesis. PI3Ks may also regulate IL1A, JUND, FOSL2, and PPP3CA through the MAPK signaling pathway. The discovery of these molecular mechanisms associated with the progression of osteoarthritis may contribute to the early diagnosis and treatment of osteoarthritis. Further work should evaluate the utility of these DEGs as biomarkers with diagnostic efficiency, as well as their potential as novel drug targets for the treatment of OA.

\section{Methods}

5.1. Research Subjects. The research subjects consisted of 19 OA patients and 22 healthy controls. All the research subjects were collected from Department of Rheumatology and Immunology, the second Xiangya Hospital of Central South University. The symptomatic primary OA patients who met the inclusion criteria were diagnosed by American College of Rheumatology(ACR) standard diagnostic criteria [34], with the Kellgren and Lawrence scoring system rating over grade 1 [35]. The Kellgren and Lawrence scoring system is divided into five grades: grade 0 means normal radiograph; grade 1 means suspicious pathology; grade 2 means small osteophytes and possible joint space narrowing; grade 3 means moderate osteophytes, definite joint space narrowing, and some of subchondral bone sclerosis; and grade 4 means large osteophytes, severe joint 


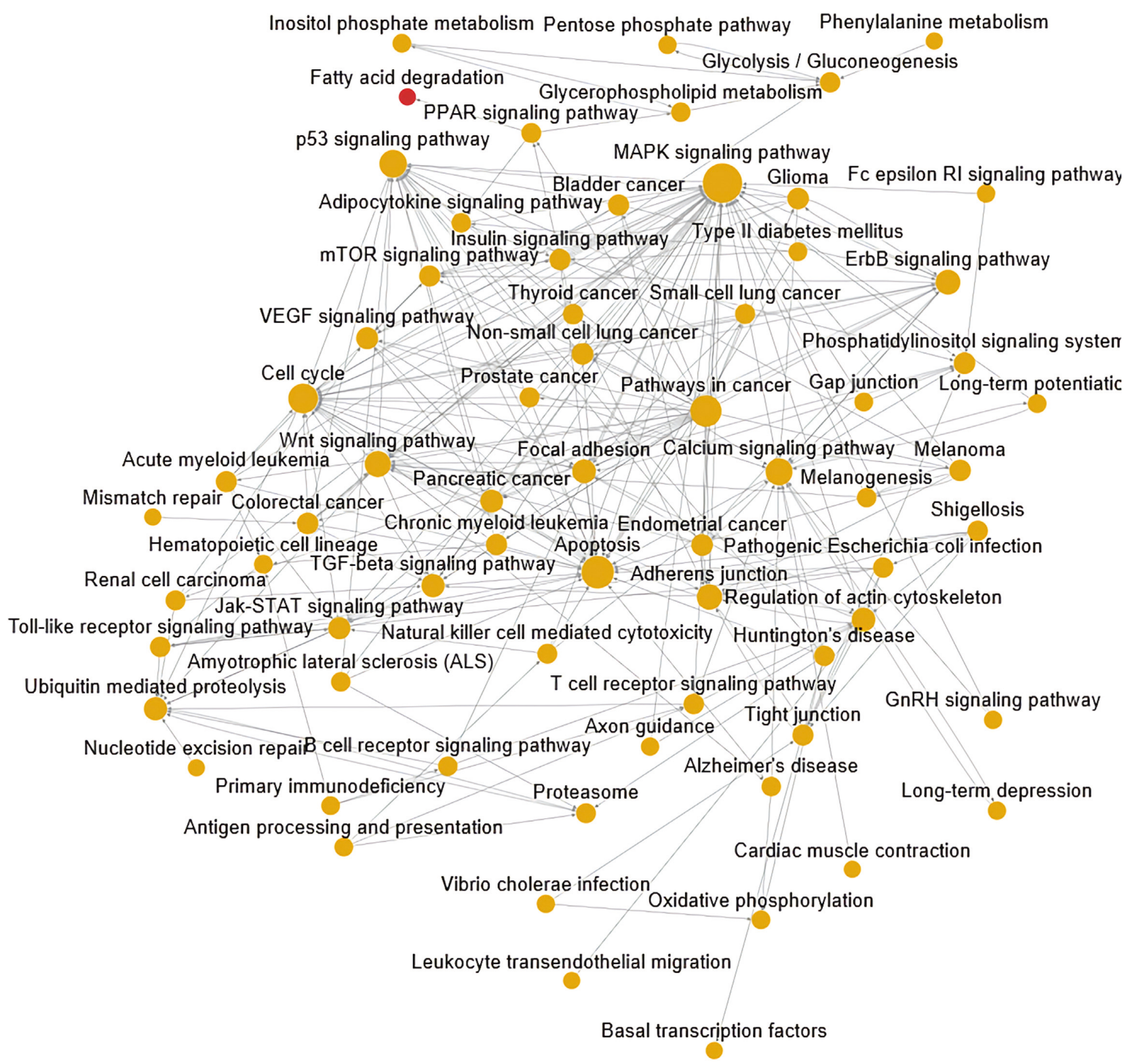

FIGURE 4: The map of signaling pathway relationship. Yellow nodes indicate the signaling pathways involved by up- and downregulated DEGs. Red nodes indicate the signaling pathways involved by upregulated DEGs. The larger the nodes, the more important they are in the network. The solid line with arrows is used to represent relationship between signaling pathways. The beginning of arrow is the upstream signaling pathway, and the end of arrows is the downstream signaling pathway.

space narrowing, and obvious subchondral bone sclerosis. Within the 19 OA patients analyzed, there were 3 OA patients of grade 1, 4 OA patients of grade 2, 6 OA patients of grade 3 , and $6 \mathrm{OA}$ patients of grade 4 . Exclusion criteria included the diagnosis of RA, osteoporosis, ankylosing spondylitis, neoplastic disease, or any other inflammatory autoimmune diseases. The healthy controls were collected from the same period of physical examination without clinical and radiographic osteoarthritis according to ACR criteria. The age, height, weight, and sex of OA patients and healthy controls were comparable. The research process followed the ethical standards of human experiment in Xiangya Medical College of Central South University and obtained the consent of the research subjects.
5.2. Monocyte Extraction and RNA Isolation. We collected fresh peripheral blood from each subject and separated circulating mononuclear cells (MNCs) with UNI-SEP separation tube (Novamed INC, Illinois, USA) according to manufacturer's recommendations. UNI-SEP products are sterile plastic centrifuge tubes containing a solution of $5.6 \%$ polysucrose and $9.6 \%$ sodium metrizoate. Then, we used monocyte negative isolation kit (Dynal Biotech ASA, Oslo, Norway) to isolate monocytes from MNCs. The antibody mix contains mouse IgG antibodies for CD2, CD7, CD16, CD19, CD56 and CD235a bound the surface of depletion dynabeads, and we discarded beads with unwanted cells and collected untouched monocytes. Then, the monocytes were lysed in TRIzol Reagent (Life Technologies, Bleiswijk, The Netherlands) for $5 \mathrm{~min}$, 


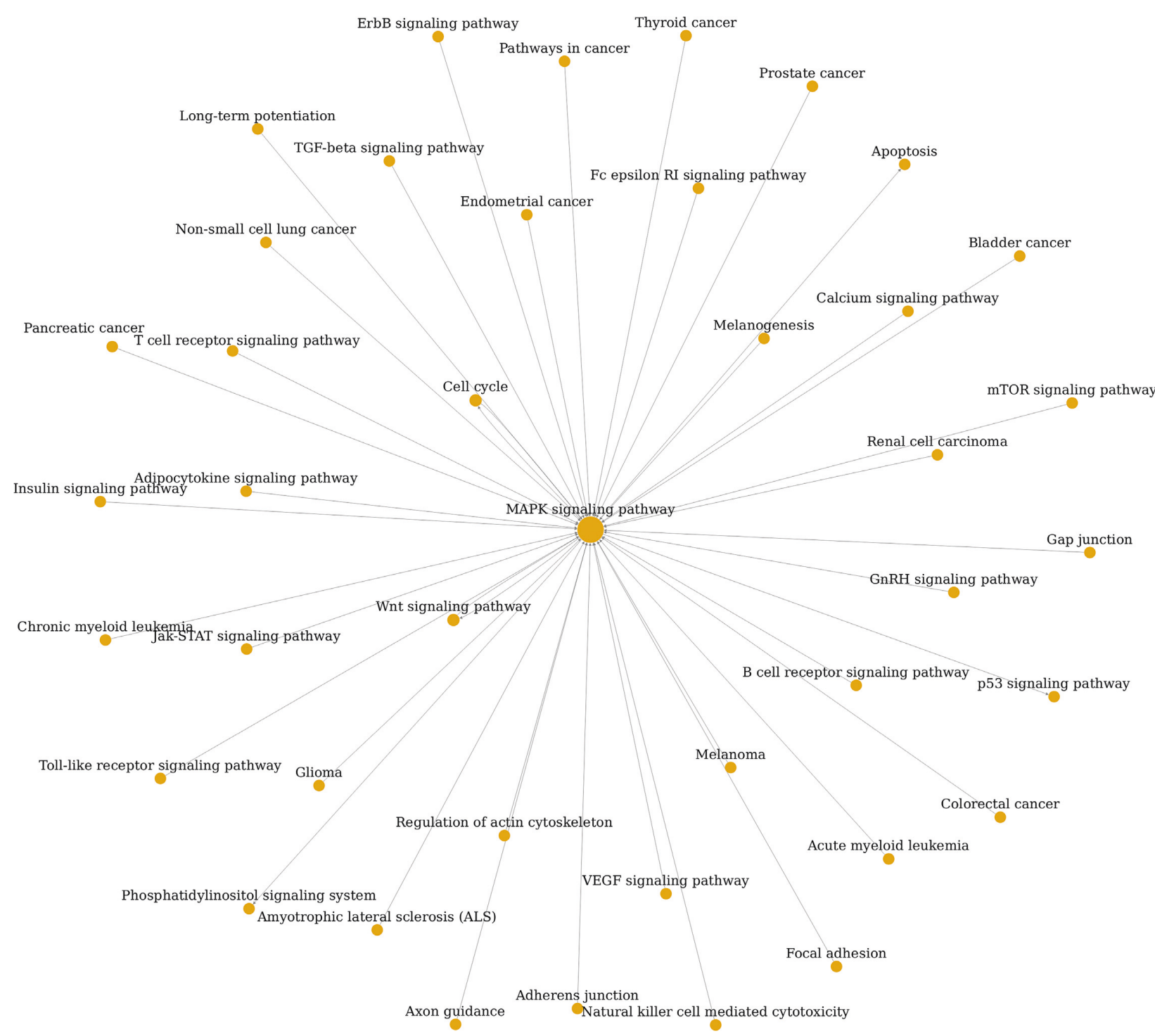

FIGURE 5: The solar map of all associated signaling pathways of the MAPK signaling pathway.

chloroform was added, and then centrifuged $(15 \mathrm{~min}$ at $12000 \mathrm{~g})$. Transferring the upper aqueous phase to another RNase-free EP tube and adding isopropanol, the tube was placed stably for $10 \mathrm{~min}$ and then centrifuged $(15 \mathrm{~min}$ at $12000 \mathrm{~g}$ ). The sediments (RNA) were washed with 75\% ethanol and dissolved in DEPC-treated water. Quality and quantity of the isolated RNA were measured by analyzing samples using the 2100 Bioanalyzer (Agilent Technologies, Amstelveen, The Netherlands) and Nanodrop spectrophotometer.

5.3. Microarray Analysis. Total RNA was reverse transcribed to synthesis first-strand cDNA by using a T7-Oligao (dT) Promoter Primer, and then second-strand cDNA was synthesized and purified. Next, in in vitro transcription reaction, second-strand cDNA serves as a template performed for complementary RNA (cRNA) amplification and biotin labeling and was cleaned up and fragmented. All the operations were following the instructions of Message Amp ${ }^{\mathrm{TM}}$ Premier RNA Amplification kit (Invitrogen, USA).
Subsequently, the biotinylated cRNAs were hybridized to Affymetrix Human Genome U133A genechip overnight at $45^{\circ} \mathrm{C}$ with rocking. Genechip was then washed and stained according to the protocol of GeneChip ${ }^{\circledR}$ Hybridization, Wash, and Stain kit (Thermo Fisher Scientific, USA). Finally, Affymetrix Gene Array Scanner G2500A scanned the chip, and the scanned images were analyzed by MAS5.0 software.

5.4. Screening of DEGs. The image signals of the chip were transformed into the original data of gene expression level by MAS5.0 software. OA group and control group were set up on the GCBI working platform (https://www.gcbi.com.cn/, GMINIX Informatics Ltd. Co, Shanghai, P.R. China). The original data CEL files were imported into the two groups. Using SAM (significance analysis of microarray) R program [36], DEGs between OA group and control group were screened according to the screening criteria of $Q<0.05$, $P<0.05$, and fold change $>1.2(Q$ value is the false discovery rate, which represents the expected proportion of false- 
positive genes in differentially expressed genes. $P$ value is adjusted by the $Q$ value. When $Q<0.05$, it can assure lower false discovery rate and higher reliability of results). Hierarchical clustering (a heatmap) was performed with these screened DEGs to identify whether they can make an obvious distinction between $\mathrm{OA}$ samples and control samples.

5.5. GO Analysis and Pathway Enrichment Analysis of DEGs. We also performed GO (gene ontology) analysis $(P<0.05$, FDR, false discovery rate $<0.05)$ and pathway enrichment analysis $(P<0.05)$ for DEGs on the GCBI working platform. In GO analysis, biological processes, cell components, and molecular processes are mainly involved. In the screening of DEGs, more information is provided by biological processes. Therefore, in GO analysis on the GCBI platform, biological processes should be selected as the "analysis type" in parameter setting.

5.6. Network Analysis. For DEGs and its associated signaling pathways, we did geneSignalNetwork and pathwayRelationNetwork analysis on the GCBI platform, so the interaction relationship of DEGs and signaling pathways was obtained.

\section{Abbreviations}

OA: Osteoarthritis

PBMCs: Peripheral blood mononuclear cells

IL-1RI: Interleukin 1 receptor type I

MMP13: Matrix metallopeptidase 13

DEGs: Differentially expressed genes

GO: Gene ontology

GCBI: Gene-Cloud of Biotechnology Information

ACR: American College of Rheumatology

RA: $\quad$ Rheumatoid arthritis

RPL38: Ribosomal protein L38

PPP3CA: Protein phosphatase 3, catalytic subunit, alpha isozyme

IKZF1: IKAROS family zinc finger 1

C7orf43: Chromosome 7 open reading frame 43

IL1A: Interleukin 1, alpha

IL6R: Interleukin 6 receptor

IL7R: $\quad$ Interleukin 7 receptor

PIK3: $\quad$ Phosphoinositide-3-kinase

MAPK14: Mitogen-activated protein kinase 14

TNFSF10: Tumor necrosis factor (ligand) superfamily, member 10

TNFSF13: Tumor necrosis factor (ligand) superfamily, member 13

ATF2: $\quad$ Activating transcription factor 2

TYK2: $\quad$ Tyrosine kinase 2

PIK3CA: Phosphatidylinositol-4,5-bisphosphate 3-kinase, catalytic subunit alpha

PIK3CB: Phosphatidylinositol-4,5-bisphosphate 3-kinase, catalytic subunit beta

PIK3CD: Phosphatidylinositol-4,5-bisphosphate 3-kinase, catalytic subunit delta

PIK3R1: $\quad$ Phosphoinositide-3-kinase, regulatory subunit 1 (alpha);
MAPK14: Mitogen-activated protein kinase 14

JUND: Jun D proto-oncogene

FOSL2: $\quad$ FOS-like antigen 2.

\section{Data Availability}

The data used to support the findings of this study are available from the corresponding author upon request.

\section{Ethical Approval}

This research process followed the ethical standards of human experiment in Xiangya Medical College of Central South University and obtained the consent of the research subjects.

\section{Conflicts of Interest}

The authors declare that they have no conflicts of interest.

\section{Authors' Contributions}

TS collected samples of research subjects and completed all microarray-related experiments. XJS performed the data analyses. GG and TS helped perform the analysis with constructive discussions. TS and XJS wrote the manuscript. All authors read and approved the final manuscript.

\section{Acknowledgments}

The authors would like to acknowledge the online analysis platform GCBI for providing us technical assistance. This work was supported by the Hunan Provincial Natural Science Fund (grant no. 2016JJ4053).

\section{References}

[1] G. Musumeci, M. A. Szychlinska, and A. Mobasheri, "Agerelated degeneration of articular cartilage in the pathogenesis of osteoarthritis: molecular markers of senescent chondrocytes," Histology and Histopathology, vol. 30, no. 1, pp. 1-12, 2015.

[2] B. Xia, D. Chen, J. Zhang, S. Hu, H. Jin, and P. Tong, "Osteoarthritis pathogenesis: a review of molecular mechanisms," Calcified Tissue International, vol. 95, no. 6, pp. 495-505, 2014.

[3] M. J. Thomas, G. Peat, T. Rathod et al., "The epidemiology of symptomatic midfoot osteoarthritis in community-dwelling older adults: cross-sectional findings from the clinical assessment study of the foot," Arthritis Research \& Therapy, vol. 17, no. 178, pp. 1-11, 2015.

[4] F. Russo, M. D’Este, G. Vadala et al., "Platelet rich plasma and hyaluronic acid blend for the treatment of osteoarthritis: rheological and biological evaluation," PLoS One, vol. 11, no. 6, Article ID e015704, 2016.

[5] S. Warner and A. Valdes, "The genetics of osteoarthritis: a review," Journal of Functional Morphology and Kinesiology, vol. 1, no. 1, pp. 140-153, 2016.

[6] W. Wang, Y. Liu, J. Hao et al., "Comparative analysis of gene expression profiles of hip articular cartilage between nontraumatic necrosis and osteoarthritis," Gene, vol. 591, no. 1, pp. 43-47, 2016.

[7] I. Prasadam, J. Batra, S. Perry, W. Gu, R. Crawford, and Y. Xiao, "Systematic identification, characterization and target 
gene analysis of microRNAs involved in osteoarthritis subchondral bone pathogenesis," Calcified Tissue International, vol. 99, no. 1, pp. 43-55, 2016.

[8] R. Park and J. D. Ji, "Unique gene expression profile in osteoarthritis synovium compared with cartilage: analysis of publicly accessible microarray datasets," Rheumatology International, vol. 36, no. 6, pp. 819-827, 2016.

[9] M. Durand, S. V. Komarova, A. Bhargava et al., "Monocytes from patients with osteoarthritis display increased osteoclastogenesis and bone resorption: the in vitro osteoclast differentiation in arthritis study," Arthritis \& Rheumatism, vol. 65, no. 1, pp. 148-158, 2013.

[10] P. Castrogiovanni, M. D. Rosa, C. Guglielmino et al., "Moderate physical activity as a prevention method for knee osteoarthritis and the role of synoviocytes as biological key," International Journal of Molecular Sciences, vol. 20, no. 3, 2019.

[11] H. Raghu, C. M. Lepus, Q. Wang et al., "CCL2/CCR2, but not CCL5/CCR5, mediates monocyte recruitment, inflammation and cartilage destruction in osteoarthritis," Annals of the Rheumatic Diseases, vol. 76, no. 5, pp. 914-922, 2017.

[12] S. A. Miller, A. J. Brown, M. C. Farach-Carson, and C. B. KirnSafran, "HIP/RPL29 down-regulation accompanies terminal chondrocyte differentiation," Differentiation, vol. 71 , no. 6 , pp. 322-336, 2003.

[13] S. S. Jiang, C.-H. Chen, K.-Y. Tseng et al., "Gene expression profiling suggests a pathological role of human bone marrowderived mesenchymal stem cells in aging-related skeletal diseases," Aging, vol. 3, no. 7, pp. 672-684, 2011.

[14] H. Green, A. E. Canfield, M. C. Hillarby et al., "The Ribosomal protein QM is expressed differentially during vertebrate endochondral bone development," Journal of Bone and Mineral Research, vol. 15, no. 6, pp. 1066-1075, 2000.

[15] J. Geurts, A. Patel, M. T. Hirschmann et al., "Elevated marrow inflammatory cells and osteoclasts in subchondral osteosclerosis in human knee osteoarthritis," Journal of Orthopaedic Research, vol. 34, no. 2, pp. 262-269, 2016.

[16] D. S. Amarasekara, H. Yun, S. Kim, N. Lee, H. Kim, and J. Rho, "Regulation of osteoclast differentiation by cytokine networks," Immune Network, vol. 18, no. 1, p. e8, 2018.

[17] D. A. Fruman, H. Chiu, B. D. Hopkins, S. Bagrodia, L. C. Cantley, and R. T. Abraham, "The PI3K pathway in human disease," Cell, vol. 170, no. 4, pp. 605-635, 2017.

[18] W. Xuan, X. Feng, C. Qian et al., "Osteoclast differentiation gene expression profiling reveals chemokine CCL4 mediates RANKL-induced osteoclast migration and invasion via PI3K pathway," Cell Biochemistry and Function, vol. 35, no. 3, pp. 171-177, 2017.

[19] S. E. Lee, K. M. Woo, S. Y. Kim et al., "The phosphatidylinositol 3-kinase, p38, and extracellular signal-regulated kinase pathways are involved in osteoclast differentiation," Bone, vol. 30, no. 1, pp. 71-77, 2002.

[20] D. Győri, D. Csete, S. Benkő et al., “The phosphoinositide 3kinase isoform $\mathrm{PI} 3 \mathrm{~K} \beta$ regulates osteoclast-mediated bone resorption in humans and mice," Arthritis \& Rheumatology, vol. 66, no. 8, pp. 2210-2221, 2014.

[21] A. Papoudou-Bai, E. Hatzimichael, A. Barbouti, and P. Kanavaros, "Expression patterns of the activator protein-1 (AP-1) family members in lymphoid neoplasms," Clinical and Experimental Medicine, vol. 17, no. 3, pp. 291-304, 2017.

[22] K. Matsuo, J. M. Owens, M. Tonko, C. Elliott, T. J. Chambers, and E. F. Wagner, "Fosll is a transcriptional target of c-Fos during osteoclast differentiation," Nature Genetics, vol. 24, no. 2, pp. 184-187, 2000.
[23] G. E. Beranger, D. Momier, J.-M. Guigonis, M. Samson, G. F. Carle, and J.-C. Scimeca, "Differential binding of pol$\mathrm{y}$ (ADP-Ribose) polymerase-1 and JunD/Fra2 accounts for RANKL-induced Tcirg1 gene expression during osteoclastogenesis," Journal of Bone and Mineral Research, vol. 22, no. 7, pp. 975-983, 2007.

[24] S. Wijekoon, E. C. Bwalya, J. Fang, S. Kim, K. Hosoya, and M. Okumura, "Chronological differential effects of pro-inflammatory cytokines on RANKL-induced osteoclast differentiation of canine bone marrow-derived macrophages," Journal of Veterinary Medical Science, vol. 79, no. 12, pp. 2030-2035, 2017.

[25] T. Mizuguchi, M. Nakashima, M. Kato et al., "Loss-offunction and gain-of-function mutations in PPP3CA cause two distinct disorders," Human Molecular Genetics, vol. 27, no. 8, pp. 1421-1433, 2018.

[26] P. N. Gabrovska, R. A. Smith, L. M. Haupt, and L. R. Griffiths, "Investigation of two Wnt signalling pathway single nucleotide polymorphisms in a breast cancer-affected Australian population," Twin Research and Human Genetics, vol. 14, no. 6, pp. 562-567, 2011.

[27] C. Hundhausen, C. Boesch-Saadatmandi, N. Matzner et al., "Ochratoxin a lowers mRNA levels of genes encoding for key proteins of liver cell metabolism," Cancer Genomics Proteomics, vol. 5, no. 6, pp. 319-332, 2008.

[28] Y. Sun, W.-Z. Liu, T. Liu, X. Feng, N. Yang, and H.-F. Zhou, "Signaling pathway of MAPK/ERK in cell proliferation, differentiation, migration, senescence and apoptosis," Journal of Receptors and Signal Transduction, vol. 35, no. 6, pp. 600-604, 2015.

[29] C. Zhang, L. Yu, Y. Zhou, Q. Zhao, and S.-Q. Liu, "Chitosan oligosaccharides inhibit IL-1 $\beta$-induced chondrocyte apoptosis via the P38 MAPK signaling pathway," Glycoconjugate Journal, vol. 33, no. 5, pp. 735-744, 2016.

[30] H. Lim and H. P. Kim, "Matrix metalloproteinase-13 expression in IL- $1 \beta$-treated chondrocytes by activation of the p38 MAPK/c-Fos/AP-1 and JAK/STAT pathways," Archives of Pharmacal Research, vol. 34, no. 1, pp. 109-117, 2011.

[31] H. Joos, W. Albrecht, S. Laufer, and R. E. Brenner, "Differential effects of p38MAP kinase inhibitors on the expression of inflammation-associated genes in primary, interleukin- $1 \beta$ stimulated human chondrocytes," British Journal of Pharmacology, vol. 160, no. 5, pp. 1252-1262, 2010.

[32] K. Lee, Y. H. Chung, H. Ahn, H. Kim, J. Rho, and D. Jeong, "Selective regulation of MAPK signaling mediates RANKLdependent osteoclast differentiation," International Journal of Biological Sciences, vol. 12, no. 2, pp. 235-245, 2016.

[33] M. Attur, I. Belitskaya-Lévy, C. Oh et al., "Increased interleukin- $1 \beta$ gene expression in peripheral blood leukocytes is associated with increased pain and predicts risk for progression of symptomatic knee osteoarthritis," Arthritis \& Rheumatism, vol. 63, no. 7, pp. 1908-1917, 2011.

[34] A. K. Singh, M. Kalaivani, A. Krishnan, P. K. Aggarwal, and S. K. Gupta, "Prevalence of osteoarthritis of knee among elderly persons in urban slums using american college of rheumatology (ACR) criteria," Journal of Clinical and Diagnostic Research, vol. 8, no. 9, pp. 9-11, 2014.

[35] J. H. Kellgren and J. S. Lawrence, "Radiological assessment of osteo-arthrosis," Annals of the Rheumatic Diseases, vol. 16, no. 4, pp. 494-502, 1957.

[36] V. G. Tusher, R. Tibshirani, and G. Chu, "Significance analysis of microarrays applied to the ionizing radiation response," Proceedings of the National Academy of Sciences, vol. 98, no. 9, pp. 5116-5121, 2001. 


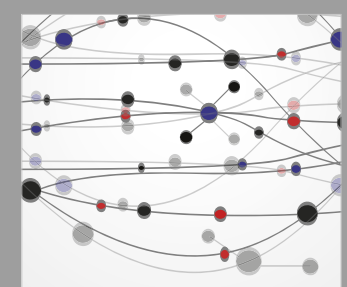

The Scientific World Journal
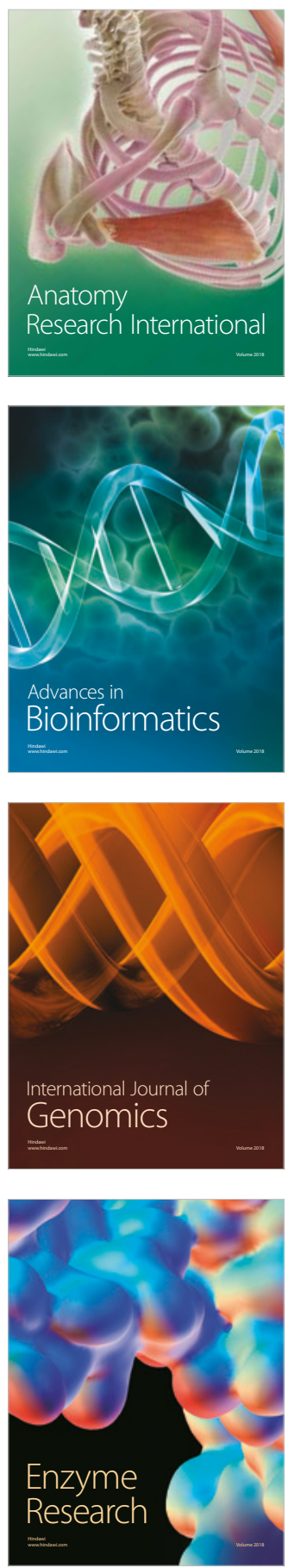
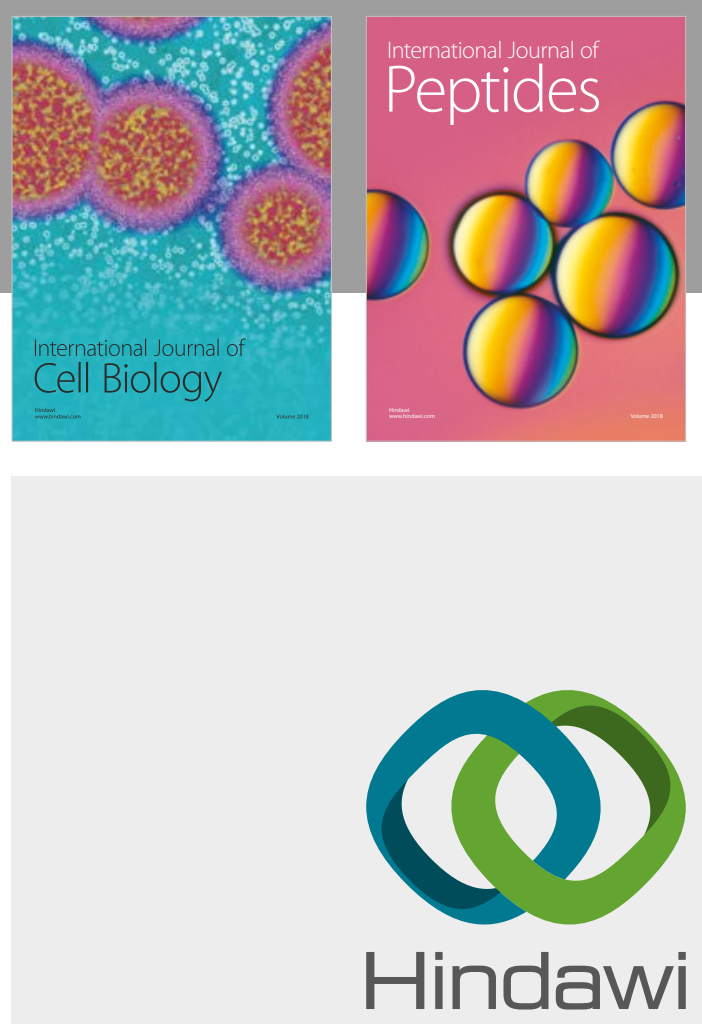

Submit your manuscripts at

www.hindawi.com
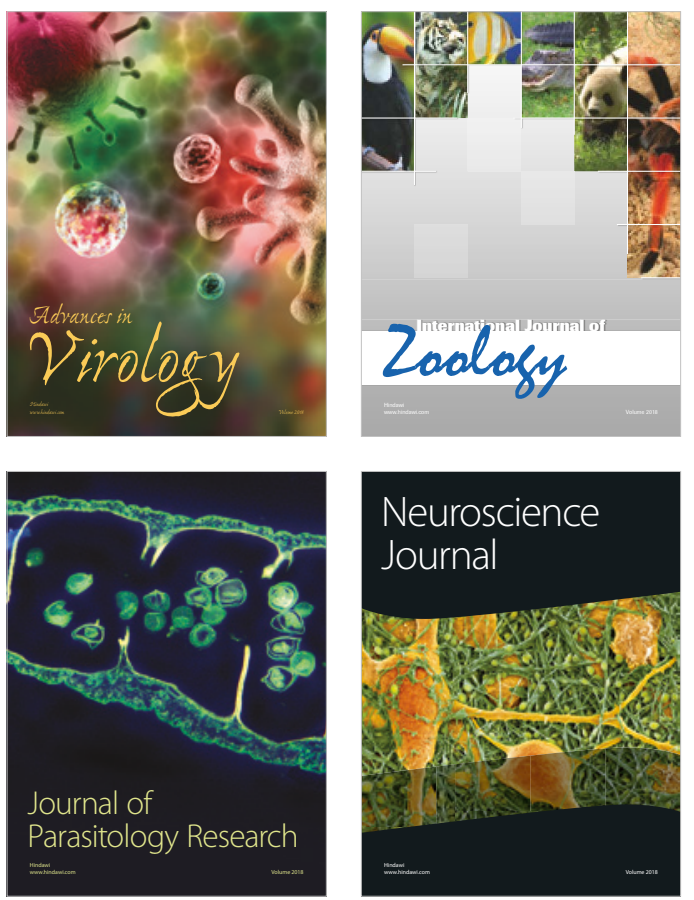
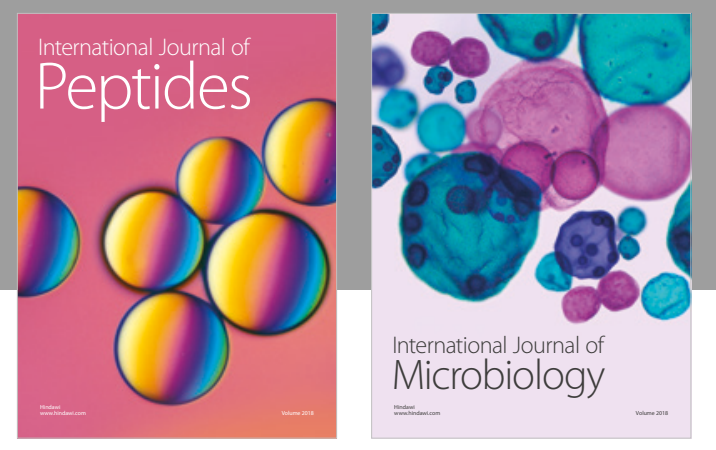

nternational Journal of Microbiology
Journal of
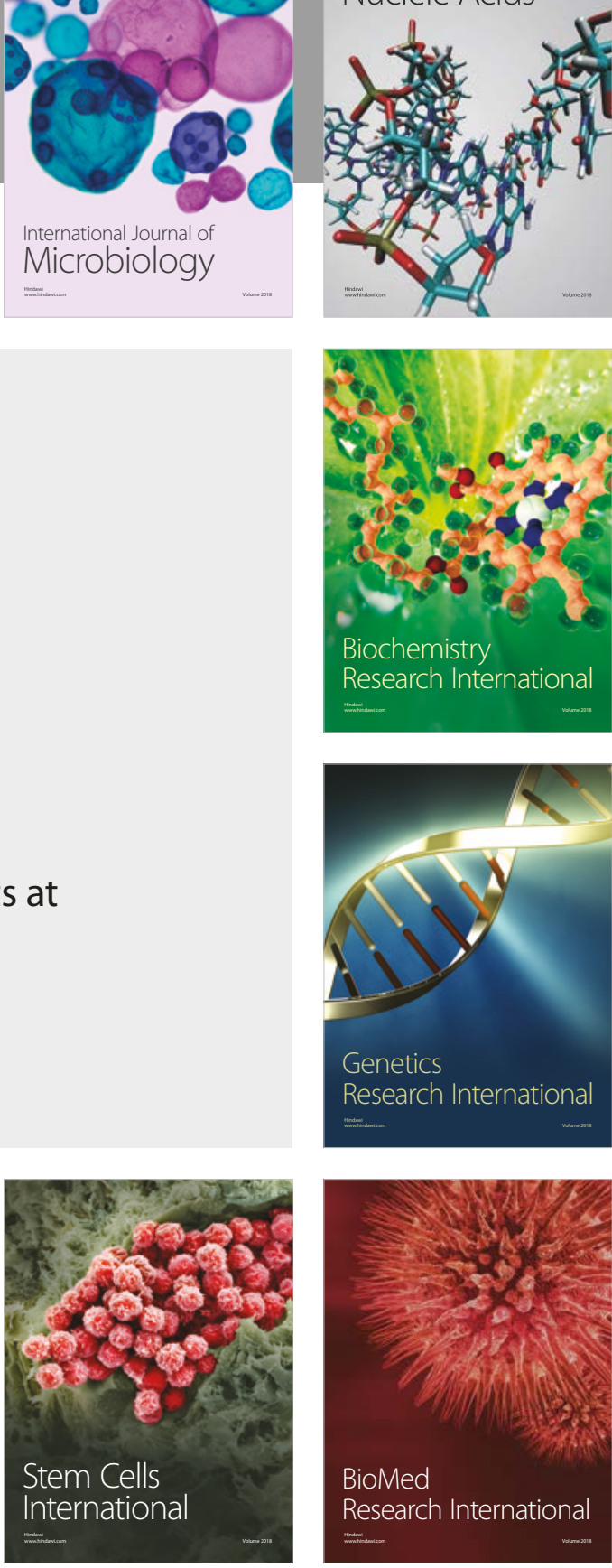
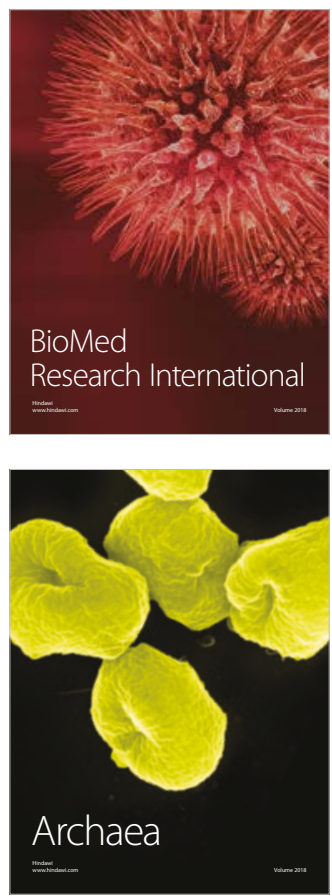